\title{
Water dynamics in different biochar fractions
}

\author{
Pellegrino Conte ${ }^{a *}$ and Nikolaus Nestle ${ }^{b}$
}

\begin{abstract}
Biochar is a carbonaceous porous material deliberately applied to soil to improve its fertility. The mechanisms through which biochar acts on fertility are still poorly understood. The effect of biochar texture size on water dynamics was investigated here in order to provide information to address future research on nutrient mobility towards plant roots as biochar is applied as soil amendment. A poplar biochar has been stainless steel fractionated in three different textured fractions $(1.0-2.0 \mathrm{~mm}, 0.3-$ $1.0 \mathrm{~mm}$ and $<0.3 \mathrm{~mm}$, respectively). Water-saturated fractions were analyzed by fast field cycling (FFC) NMR relaxometry. Results proved that 3D exchange between bound and bulk water predominantly occurred in the coarsest fraction. However, as porosity decreased, water motion was mainly associated to a restricted 2D diffusion among the surface-site pores and the bulk-site ones. The X-ray $\mu$-CT imaging analyses on the dry fractions revealed the lowest surface/volume ratio for the coarsest fraction, thereby corroborating the 3D water exchange mechanism hypothesized by FFC NMR relaxometry. However, multi-micrometer porosity was evidenced in all the samples. The latter finding suggested that the 3D exchange mechanism cannot even be neglected in the finest fraction as previously excluded only on the basis of NMR relaxometry results. X-ray $\mu$-CT imaging showed heterogeneous distribution of inorganic materials inside all the fractions. The mineral components may contribute to the water relaxation mechanisms by FFC NMR relaxometry. Further studies are needed to understand the role of the inorganic particles on water dynamics. Copyright $\odot 2015$ John Wiley \& Sons, Ltd.
\end{abstract}

Keywords: FFC NMR relaxometry; micro-CT imaging; micro-tomography; biochar; texture size; water; dynamics

\section{Introduction}

Biochar (BC) is a carbonaceous porous material obtained by pyrolysis of biomasses. It shows great potential in improving soil fertility. ${ }^{[1-6]}$ Notwithstanding the amount of studies dealing with biochar role in soil fertility, the mechanisms of its action in environment are still poorly understood.

One of the main concerns in biochar physical chemistry is its water holding capacity (WHC) and its ability to increase soil WHC as it is used as amendment. ${ }^{[3]}$ In particular, porosity plays the major role in retaining different amounts of water in biochar and biocharcontaining systems. Recently, Conte et al. ${ }^{[7]}$ reported that water in biochar undergoes 2D surface diffusion as it is constrained in micropores. In fact, water fugacity towards the bulk liquid is inhibited by the strong interactions with the pore walls. Conversely, as pore size enlarges, the amount of water exchanging with the bulk increases as a consequence of the shorter residence time of water on the pore walls. Similar results have been reported in Baiamonte et al. ${ }^{[6]}$ These authors investigated soil structure alterations as a consequence of biochar amendments. Namely, water retention in biochar amended soils appeared mainly affected by intra-aggregate porosity (i.e. pores spaces between particles within the fabric units, textural pores, micropores) as the amount of biochar was below 0.33 (this is the fraction of biochar, $f_{\mathrm{bc}}$, calculated by dividing the amount of carbonaceous pore material by the sum of the amount of biochar and that of the soil). Conversely, water retention was influenced by the swelling processes driven by inter-aggregate porosity (i.e. pore spaces between fabric units, structural pores, macropores) as $f_{\mathrm{bc}}$ overcame the limit value of 0.33 . $^{[6]}$

The chemical mechanisms of water retention in biochar (or in biochar amended soils) have been clarified in Conte et al. ${ }^{[8]}$ Here, the authors stated that water may interact with ashes and polycondensed aromatic rings through interactions mediated either by the electron deficiencies of the metals in the biochar inorganic constituents or by the $\pi$-clouds of the organic system. In fact, electron-deficient metals may form charge-transfer bindings with the electron-rich oxygen present in water, whereas unconventional $\mathrm{H}$-bonds occur via electron donation from the $\pi$-clouds of the poly-condensed aromatic systems towards the electron-deficient hydrogen in water.

Up to now, the role played by porosity in WHC of biochar has been investigated only on materials obtained either by different feedstocks or by different production procedures. ${ }^{[7,9]}$ No information have been provided yet on water dynamics in biochar porous systems differing only in terms of their texture size (i.e. fractions achieved by sieving a biochar with different sized sieves). On the bases of the aforementioned water dynamics mechanisms, it can be expected that water is subjected mainly to $2 \mathrm{D}$ diffusion once it is included into the smallest textured biochar fraction. As water is mainly located inside larger inter-particle pores in the largest textured biochar fraction, predominance of the aforementioned 3D exchange mechanisms may occur. Finally, co-occurrence of both 2D and $3 \mathrm{D}$ motions takes place as water resides inside biochar fractions with an average sized texture.

In the present study, we have fractionated the poplar biochar already analyzed in De Pasquale et al. ${ }^{[9]}$ and Conte et al. ${ }^{[7,8]}$ in three

\footnotetext{
* Correspondence to: Pellegrino Conte, Dipartimento di Scienze Agrarie e Forestali, Università degli Studi di Palermo, 90128 Palermo, Italy. E-mail: pellegrino. conte@unipa.it

a Dipartimento di Scienze Agrarie e Forestali, Università degli Studi di Palermo, 90128, Palermo, Italy
}

b BASF SE Advanced Materials and Systems Research, Ludwigshafen, Germany 
different texture sized fractions (i.e. $<0.3,0.3-1.0$ and $1.0-2.0 \mathrm{~mm}$ ). Each fraction was suspended in water and analyzed by fast field cycling (FFC) NMR relaxometry in order to validate the $2 \mathrm{D}$ and $3 \mathrm{D}$ diffusion mechanisms hypothesized in the preceding texts. The dried texture fractions were also investigated by X-ray $\mu-C T$ imaging to provide images of the spaces available to water dynamics. Combination of FFC NMR relaxometry and X-ray $\mu-C T$ imaging showed not only that there is a correlation between biochar surface chemical composition and pore sizes but also that the 3D exchange mechanism appears to predominate in all the fractions regardless of their pore sizes.

\section{Materials and Methods}

\section{Biochar sample}

Poplar (Populus spp. L.) wood chips were collected by cutting dedicated short rotation forestry in the Po Valley (Gadesco Pieve Delmona, $\left.45^{\circ} 10 \mathrm{I} 13 \mathrm{IIN}, 10^{\circ} 06 \mathrm{I01} \mathrm{IIE}\right)$ when the age of the forestry was 5 years. The wood chips were used for the gasification process described in De Pasquale et al. ${ }^{[9]}$ to retrieve syngas and biochar. All the biochar analyses have been already reported in De Pasquale et al. ${ }^{[9]}$

\section{Sample preparation}

Biochar was oven-dried overnight at $40^{\circ} \mathrm{C}$ then sieved in three texture fractions. The first fraction, indicated as $1.0-2.0 \mathrm{~mm}$, was obtained by using a $2.0 \mathrm{~mm}$ stainless steel sieve. The second $0.3-1.0 \mathrm{~mm}$ fraction was retrieved by sieving the first texture fraction with a $1 \mathrm{~mm}$ sieve. Finally, the last fraction, $<0.3 \mathrm{~mm}$, was obtained by sieving the second fraction with a $0.3 \mathrm{~mm}$ sieve. It must be noticed that sieving can produce fractions that are different not only in size but also chemically. In fact, industrial biochar production involves formation of two chemically distinct fractions. The primary biochar is achieved by primary reactions occurring in the solid state, whereas secondary biochar is obtained by condensation of volatile systems that produce soot, graphene-like and tar materials. The latter shows a size much smaller than $0.3 \mathrm{~mm}$, thereby leading to the consideration that the smallest size fraction retrieved in the present study could be richer in secondary biochar. Moreover, because of its nanosize, secondary biochar can be present also in the coarsest fraction as occluding material.

\section{Fast field cycling NMR experiments}

The theory describing FFC NMR relaxometry can be found in Anoardo et al., ${ }^{[10]}$ Kimmich and Anoardo, ${ }^{[11]}$ Ferrante and Sykora. ${ }^{[12]}$ Details on the environmental application of the technique are reported in Conte and Alonzo. ${ }^{[13]}$

One gram of each texture fraction as obtained in the preceding texts was suspended in $3 \mathrm{ml}$ of deionized water and subjected to FFC NMR relaxometry investigation.

${ }^{1} \mathrm{H}$ nuclear magnetic resonance dispersion (NMRD) profiles (i.e. relaxation rates $R_{1}$ or $1 / T_{1} \mathrm{v}$. proton Larmor frequencies) were acquired on a Stelar Spinmaster FFC2000 Relaxometer (Stelar S.r.l., Mede, $\mathrm{PV}$, Italy) at $25^{\circ} \mathrm{C}$. The proton spins were polarized at a polarization field $\left(\mathrm{B}_{\mathrm{POL}}\right)$ corresponding to a proton Larmor frequency $\left(\omega_{\mathrm{L}}\right)$ of $24 \mathrm{MHz}$ for a period of polarization ( $\left.T_{\mathrm{POL}}\right)$ corresponding to about five times the $T_{1}$ estimated at this frequency. After each $\mathrm{B}_{\mathrm{POL}}$ application, the magnetic field strength (indicated as $B_{\mathrm{RLX}}$ ) was systematically changed in the proton Larmor frequency comprised in the range $0.01-40.0 \mathrm{MHz}$. The period $\tau$, during which $\mathrm{B}_{\mathrm{RLX}}$ was applied, has been varied on 32 logarithmic spaced time sets, each of them adjusted at every relaxation field in order to optimize the sampling of the decay/recovery curves. Free induction decays (FID) were recorded following a single ${ }^{1} \mathrm{H} 90^{\circ}$ pulse applied at an acquisition field $\left(B_{A C Q}\right)$ corresponding to the proton Larmor frequency of $16.2 \mathrm{MHz}$. A time domain of $100 \mu$ s sampled with 512 points was applied. Field-switching time was $3 \mathrm{~ms}$, while spectrometer dead time was $15 \mu \mathrm{s}$. For all experiments, a recycle delay of $12 \mathrm{~s}$ was used. A nonpolarized FFC sequence was applied when the relaxation magnetic fields were in the range of the proton Larmor frequencies comprised between 40.0 and $9.0 \mathrm{MHz}$. A polarized FFC sequence was applied in the proton Larmor frequencies $B_{R L X}$ range of 9.0$0.01 \mathrm{MHz}^{[11]}$

\section{FFC NMR data elaboration}

$R_{1}$ values were achieved by interpolating the ${ }^{1} \mathrm{H}$ magnetization decay/recovery curves at each $B_{R L X}$ value (i.e. ${ }^{1} \mathrm{H}$ signal intensity vs $\tau$ ) with the stretched exponential function (also known as Kohlrausch-Williams-Watts function) reported in Eqn (1) after exportation of the experimental data to OriginPro 7.5 SR6 (Version 7.5885, OriginLab Corporation, Northampton, MA, USA). The choice of this function was because of the large sample heterogeneity resulting in a multi-exponential behaviour of the decay/recovery curves. ${ }^{[14]}$ This approach has the advantage that it is able to handle a wide range of behaviours within a single model. For this reason, assumptions about the number of exponentials to be used in modelling NMRD data are not necessary.

$$
I(\tau)=I_{0} \exp \left\lfloor-\left(\tau / T_{1}\right)^{k}\right\rfloor
$$

In Eqn (1), I $(\tau)$ is the ${ }^{1} \mathrm{H}$ signal intensity at each fixed $\mathrm{B}_{\mathrm{RLX}}, I_{0}$ is the ${ }^{1} \mathrm{H}$ signal intensity at the thermal equilibrium, $T_{1}$ is the average proton spin lattice relaxation time and $k$ is a heterogeneity parameter related to the stretching of the decay process. Its value is 1 when mono-exponential decay occurs, whereas decreasing $k$ values indicate increasingly strong deviation from the mono-exponential behaviour. This function can be considered as a superposition of exponential contributions, thereby describing the likely physical picture of some distribution in $T_{1}$.

The NMRD profiles reporting the calculated $R_{1}$ values versus Larmor angular frequency $\left(\omega_{\mathrm{L}}\right)$ were exported to OriginPro 7.5 SR6 and fitted with the Bloembergen-Purcell-Pound (BPP) model modified by the model free analysis developed by Halle et al. ${ }^{[15]}$ :

$$
R_{1}=\alpha+\beta\left[0.2 \sum_{n=1}^{N} c_{n} \frac{\tau_{n}}{1+\left(\omega_{L} \tau_{n}\right)^{2}}+0.8 \sum_{n=1}^{N} c_{n} \frac{\tau_{n}}{1+\left(2 \omega_{L} \tau_{n}\right)^{2}}\right]
$$

In Eqn (2), $R_{1}$ is the longitudinal relaxation rate, $\omega_{\mathrm{L}}$ is the proton Larmor frequency, $\tau$ is the correlation time, a typical parameter for spectral density that, in turn, describes random molecular motions, $\alpha$ represents the high-field relaxation rate, while $\beta$ is a constant related to the dipolar interactions. Increasing dipolar strengths, as a result of reduced molecular mobility, produce longer $\alpha$ and larger $\beta$ values. Conversely, weak dipolar couplings are generated by unbound (or freely moving) molecules, thereby providing shorter $\alpha$ and smaller $\beta$ values. ${ }^{[9,11]}$ The number $N$ of Lorentzians that can be included in Eqn (2) without unreasonably increasing the number 
of parameters was determined by applying the $F$ test as described in Conte and Alonzo. ${ }^{[13]}$

The obtained six fit parameters $\left(c_{1}, c_{2}, c_{3}, \tau_{1}, \tau_{2}, \tau_{3}\right)$ were used to retrieve an average correlation time according to Eqn (3) ${ }^{[15]}$.

$$
\left\langle\tau_{C}\right\rangle=\frac{\sum_{n} c_{n} \tau_{n}}{\sum_{n} c_{n}}
$$

Finally, the decay curve retrieved at the proton Larmor frequency of $100 \mathrm{kHz}$ was transformed by the UPEN algorithm ${ }^{[13]}$ in order to obtain the $T_{1}$ distributions at such magnetic field and, therefore, information on the pore size distribution in each char texture class. The choice of UPEN analyses only at $100 \mathrm{kHz}$ was because of the best compromise between sensitivity and resolution. In fact, it is known that the lower the proton Larmor frequency, the larger is the number of molecular fluctuations that can be monitored by relaxometry. ${ }^{[13,16]}$ However, signal-to-noise ratio $(\mathrm{S} / \mathrm{N})$ decreases as the proton Larmor frequency is reduced. For this reason, the uncertainty in the evaluation of the distribution of $T_{1}$ by applying the UPEN algorithm increases as the magnetic field strength is decreased. ${ }^{[13]}$ The largest number of molecular fluctuations with the best $\mathrm{S} / \mathrm{N}$ ratio could be monitored with the inverse Laplace transformation of the FID acquired at $100 \mathrm{kHz}$.

\section{X-ray micro-CT}

X-ray micro-CT was performed on a Bruker Skyscan $1172 \mu \mathrm{CT}$ system (Bruker Skyscan, Antwerp, Belgium) equipped with a $10 \mathrm{~W} \mathrm{X-}$ ray generator with a maximum acceleration voltage of $100 \mathrm{kV}(\mathrm{Ha}-$ mamatsu, Hamamatsu, Japan) and an 11 MPixel X-ray camera (Ximea, Münster, Germany). For imaging the biochar samples, a voltage of $40 \mathrm{kV}$ was used and no filtering of the X-rays was performed. The biochar samples were packed into polyethylene containers of different sizes or fixed onto the sample holder with modelling wax. Depending on the sample size and the camera resolution applied, voxel sizes between 9.94 and $0.87 \mu \mathrm{m}$ were achieved in the images. Image processing was performed using the NRecon and Data Viewer tools provided by Bruker Skyscan.

\section{Results}

\section{Low-field ${ }^{1} \mathrm{H}$ NMR measurements: $T_{1}$ distributions and NMRD profiles}

Figure 1 reports the distribution of $T_{1}$ values (also referred to as relaxograms) obtained by applying the UPEN algorithm as indicated in Materials and Methods. The relaxograms show that water $T_{1}$ distribution in the largest texture size fraction (i.e. $1.0-2.0 \mathrm{~mm}$, continuous line in Fig. 1) spanned from about $20 \mathrm{~ms}$ up to about $4 \mathrm{~s}$, with two main maxima at around 50 and $900 \mathrm{~ms}$. The intermediate texture fraction (i.e. $0.3-1.0 \mathrm{~mm}$, dashed line in Fig. 1) provided a water $T_{1}$ distribution ranging in the interval $5 \mathrm{~ms}-1 \mathrm{~s}$ with maxima at 50 and $180 \mathrm{~ms}$, respectively. Finally, the lowest texture fraction ( $<0.3 \mathrm{~mm}$, dotted line in Fig. 1$)$ revealed only a maximum at $90 \mathrm{~ms}$ within a $T_{1}$ range comprised between 5 and $200 \mathrm{~ms}$.

As the magnetic field is changed within the proton Larmor frequency included in the range $40-0.01 \mathrm{MHz}$, the NMRD profiles reporting $R_{1}\left(=1 / T_{1}\right)-v s-\omega_{\mathrm{L}}$ (Fig. 2$)$ are retrieved. The three NMRD profiles are approximatively parallel to each other and translated in the order $R_{1(<0.3 \mathrm{~mm})}>R_{1(0.3-1.0 \mathrm{~mm})}>R_{1(1.0-2.0 \mathrm{~mm}) \text {, }}$ thereby indicating, as expected (refer to discussion in the succeeding texts), that relaxation rates are directly related to the sieved sample fraction.

Figure 3 reports correlation time $\left(\left\langle\tau_{C}\right\rangle\right), \alpha$ and $\beta$ values as retrieved by application of Eqn (2). It comes that all the parameters vary in the order fraction $_{(<0.3 \mathrm{~mm})}>$ fraction $_{(0.3-1.0 \mathrm{~mm})}>$ fraction $_{(1.0-2.0 \mathrm{~mm}) \text {. }}$

\section{X-ray micro-CT}

Figure 4 shows cross-section images from a first series of micro-CT scans with a voxel resolution of $9.94 \mu \mathrm{m}$. Three different grain sizes of carbon used in the NMRD experiments have been accounted for. The enlarged details in the lower row of images show a dramatic difference in the packing density between the two finer sieved fractions $(0.3-1.0 \mathrm{~mm}$ and $<0.3 \mathrm{~mm})$ and the coarsest fraction (1.0$2.0 \mathrm{~mm}$ ). Furthermore, a high degree of chemical heterogeneity is also found in all samples. In fact, a much stronger X-ray absorption differentiates the inorganic components from the neat carbon regions that can be nicely recognized by the cellular structure of the wood. The latter is essentially conserved during the charring

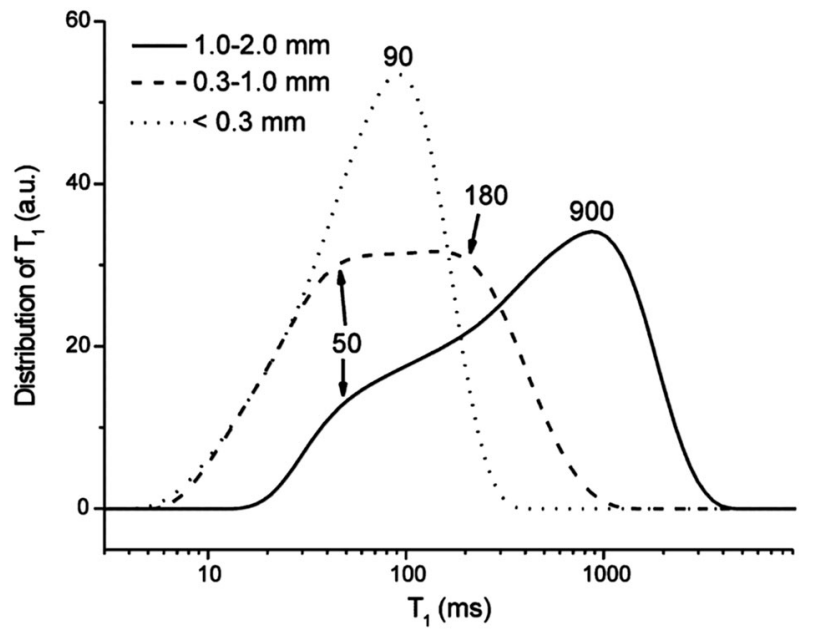

Figure 1. Relaxograms (i.e. distribution of longitudinal relaxation times) for the three texture classes $1.0-2.0 \mathrm{~mm}, 0.3-1.0$ and $<0.3 \mathrm{~mm}$.

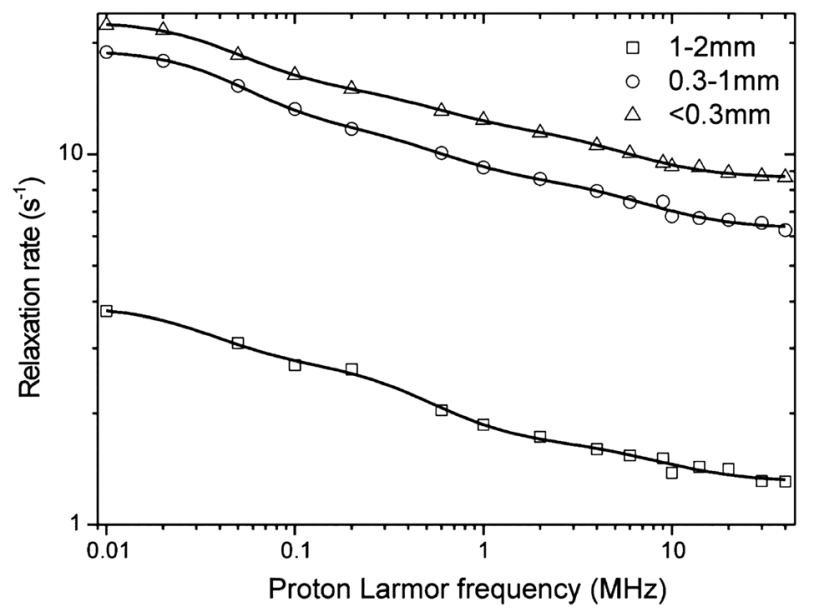

Figure 2. NMRD profiles (i.e. variations of longitudinal relaxation rates vs proton Larmor frequency) for the 1.0-2.0, $0.3-1.0$ and $<0.3 \mathrm{~mm}$ texture classes. 

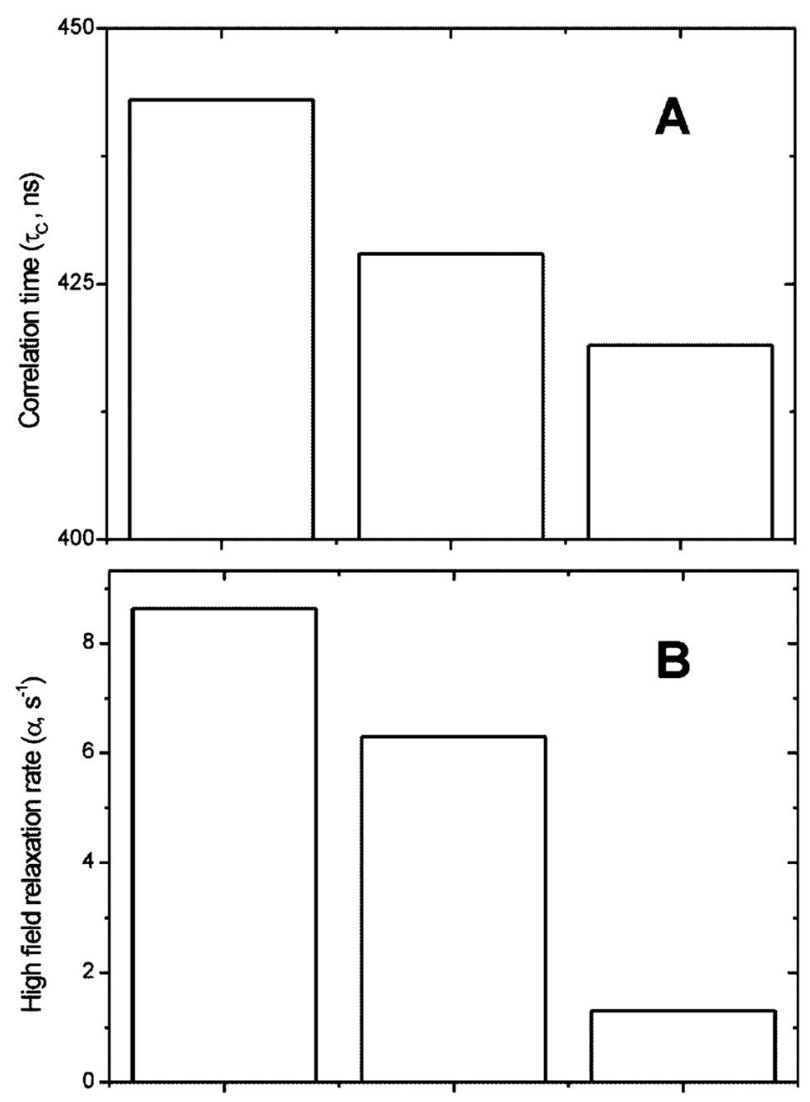

the char. Therefore, some additional experiments were conducted to better understand the nature and also the origin of the inorganic components.

Figure 6 shows a crosshair view and a cross-sectional detail of a scan of an individual carbon piece from the coarsest fraction (i.e. fraction 1.0-2.0 mm). In Fig. 7, a scan of the same piece after several days of washing in demineralized water is shown. A reduction in the fraction of inorganic material can be observed by comparing the fresh and the washed sample. The strongly inhomogeneous distribution of inorganic material over the biochar piece raises the question of the origin of the inorganics. Therefore, a fresh piece of small-diameter poplar wood (which was the feedstock for the char production) was also scanned (Fig. 8). The images in Fig. 8 show that both the bark and the inner lumen of the twig exhibit similarly strong X-ray absorption as it observed in the char specimens.

\section{Discussion}

\section{Distributions of longitudinal relaxation times}

Biochar system has been described as a porous material where two qualitatively different types of pores can be recognized: surface-site pores also indicated as $\alpha$-type and bulk-site pores referred to as $\beta$ types. ${ }^{[17]}$ As biochar is water-saturated (refer to Materials and Methods), water moves between the $\alpha$-type and $\beta$-type pores through diffusional processes that can be hampered physically by the pore sizes and chemically by the solid-liquid interactions. ${ }^{[8,17]}$ Namely, water-biochar interactions occur via formation of unconventional $\mathrm{H}$-bonds involving the electron-deficient orbitals of the hydrogen atoms in water and the $\pi$-clouds of the biochar aromatic systems. ${ }^{[8]}$ In addition, apart from the 2D diffusion between the $\alpha$ type and $\beta$-type sites, water can also escape from the biochar surface towards the bulk solution via a $3 \mathrm{D}$ exchange mechanism. ${ }^{[7]}$ Following soil pore classification, the $\alpha$-type and $\beta$-type pores can be also indicated as transmission-like $(\geq 50 \mu \mathrm{m})$, storage-like $(0.5$ $50 \mu \mathrm{m})$ and residual-like $(<0.5 \mu \mathrm{m})$ pores. ${ }^{[18,19]}$ The largest transmission-like pores are responsible for the excess water drainage, thus permitting the aforementioned water 3D exchange. Storage-like pores retain water against gravity and release, thereby allowing either a 3D exchange (from pores whose size belong to the top limit of the $0.5-50 \mu \mathrm{m}$ interval) or a $2 \mathrm{D}$ diffusion within the biochar pores. Finally, the smallest residual-like pores contain strongly bound water that cannot easily escape from the porous system.

Proton longitudinal relaxation is dominated by the presence of

Figure 3. Values of the correlation times (A), high-field longitudinal relaxation rates $(B)$ and dipolar interaction strengths $(C)$ for the $1.0-2.0$, $0.3-1.0$ and $<0.3 \mathrm{~mm}$ texture classes. All the NMRD parameters were obtained by applying the Bloembergen-Purcell-Pound model modified by the free model analysis developed by Halle et al. (1998).

process. In particular, biochar grains with conserved wood cellular structure are found in coarse and medium samples, whereas image resolution is not sufficient for visualizing cell structure in the finestgrained sample (i.e. fraction $<0.3 \mathrm{~mm}$ ). For this reason, the latter fraction was subjected to a scan with a $0.74 \mu \mathrm{m}$ resolution. Figure 5 reports a full cross section of the finest fraction and a detail view with the full voxel resolution. From these images, it appears that also fraction $<0.3 \mathrm{~mm}$ is dominated by grains with fragments of wood-like pores having sizes in the range of several micrometres.

As the micro-CT images indicate a strong presence of inorganic material in all samples, these components are likely to play also an important role in the NMR relaxation of the water contained in strong relaxation sinks at the pore surface that are because of the temporary adsorption of water on the solid surface according to the aforementioned interaction mechanisms. ${ }^{[7,8,13]}$ The effectiveness of the relaxation sinks depends on the ratio between the pore surfaces and the pore volumes as well as on the diffusion rate across pores. ${ }^{[20]}$ In particular, space restriction in small-sized pores do not allow fast molecular mobility. Once this condition occurs, ${ }^{1} \mathrm{H}-{ }^{1} \mathrm{H}$ dipolar interactions becomes very efficient and shorter relaxation times are retrieved. ${ }^{[13]}$ Conversely, as pore size enlarges, molecular mobility increases and strength of dipolar interactions weaken, thereby leading to longer relaxation times. ${ }^{[13]}$ As the number and size of the various pores becomes heterogeneous, water persists in a wide variety of differently sized pores thus providing a broad ensemble of longitudinal relaxation times that appear continuously distributed. Water molecules residing inside the smallest pores (i.e. residual-like pores) provide a range of $T_{1}$ values that is 

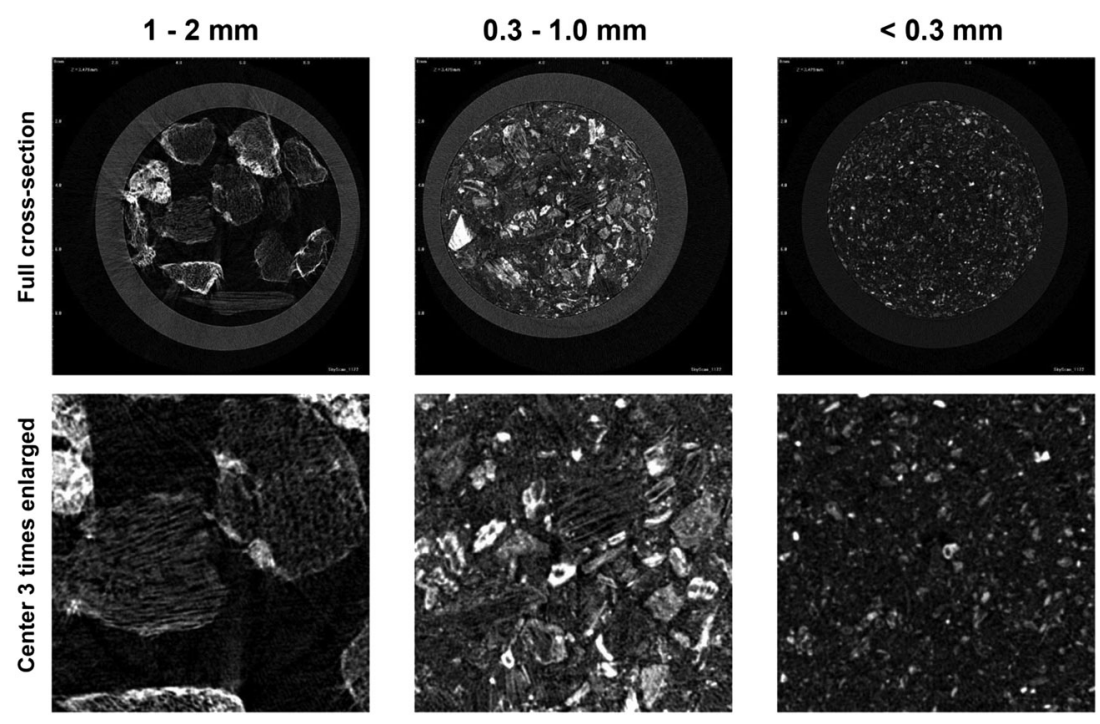

Figure 4. X-ray microtomography cross sections of the three different char texture classes. The lower row of images shows the centre of the images in threefold magnification.

close to the shortest $T_{1}$ value, whereas the $T_{1}$ values close to the longest $T_{1}$ can attributed to water located in the largest pores (i.e. transmission-like pores). All $T_{1}$ values between the two limits are because of the dynamics of water inside pores having sizes lying between the two extremes (i.e. storage-like pores) or to incomplete exchange between pores of different size.

According to the aforementioned mechanism, the differences observed in the relaxograms reported in Fig. 1 can be attributed to the different pore size distributions in the three differently textured biochar fractions. In particular, the largest band centred at $900 \mathrm{~ms}$ in the relaxogram of the $1.0-2.0 \mathrm{~mm}$ fraction (continuous line in Fig. 1) can be assigned to water freely moving in transmission-like pores, whereas the smallest band at around $50 \mathrm{~ms}$ is conceivably generated by the water molecules occluded in the residual-like pores. The continuous band between the aforementioned maxima is because of water molecules that are confined in storage-like pores.

The relaxogram of the water saturated $0.3-1.0 \mathrm{~mm}$ fraction (dashed line in Fig. 1) does not show bands in the $T_{1}$ region assigned to water moving in transmission-like pores (i.e. around $900 \mathrm{~ms}$ ). However, it shows two maxima, similar in intensity, at $180 \mathrm{~ms}$, as a result of water inside storage-like pores, and at
$50 \mathrm{~ms}$, as a result of water moving in residual-like pores. The presence of a $T_{1}$ tail in the interval $5-50$ ms indicates that the amount of residual-like pores is larger than in the coarsest biochar fraction. Moreover, the higher relaxogram intensity between 50 and $180 \mathrm{~ms}$ also accounts for a larger proportion of storage-like pores in fraction $0.3-1.0 \mathrm{~mm}$ rather than in fraction $1.0-2.0 \mathrm{~mm}$.

Water in the $<0.3 \mathrm{~mm}$ texture fraction produced the dotted relaxogram reported in Fig. 1. It consists in a homogeneous distribution of $T_{1}$ values (as compared with the other two larger textured fractions) where the most abundant pore size component seems to be the storage-like one. In fact, only one band centred at $90 \mathrm{~ms}$ appears. However, the tail in the $5-50 \mathrm{~ms} T_{1}$ range (which is similar to that observed for $0.3-1.0 \mathrm{~mm}$ fraction) suggests that a number of residual-like pore sites are also available for water molecules. Moreover, the narrower shape of the dotted relaxogram in Fig. 1, as compared with the dashed one, is an indication that there are pores having sizes nearby the lower limit of the storage-like pore size interval (i.e. $0.5-50 \mu \mathrm{m}$ ).

The comparison among the relaxograms reported in Fig. 1 indicates that the simple physical fractionation retrieved by sieving poplar biochar sample with a series of three stainless steel sieves (refer to Materials and Methods), separated three distinct types of
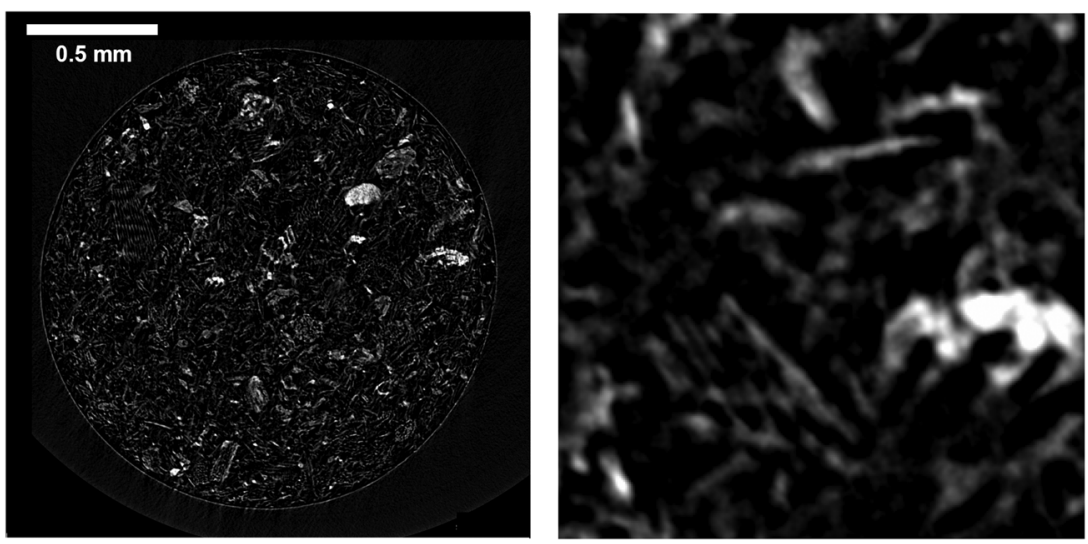

Figure 5. High-resolution microtomograph of the finest sieved fraction. On the right hand side, a 10-times enlarged detail view from the centre of the image on the left hand side is given. 

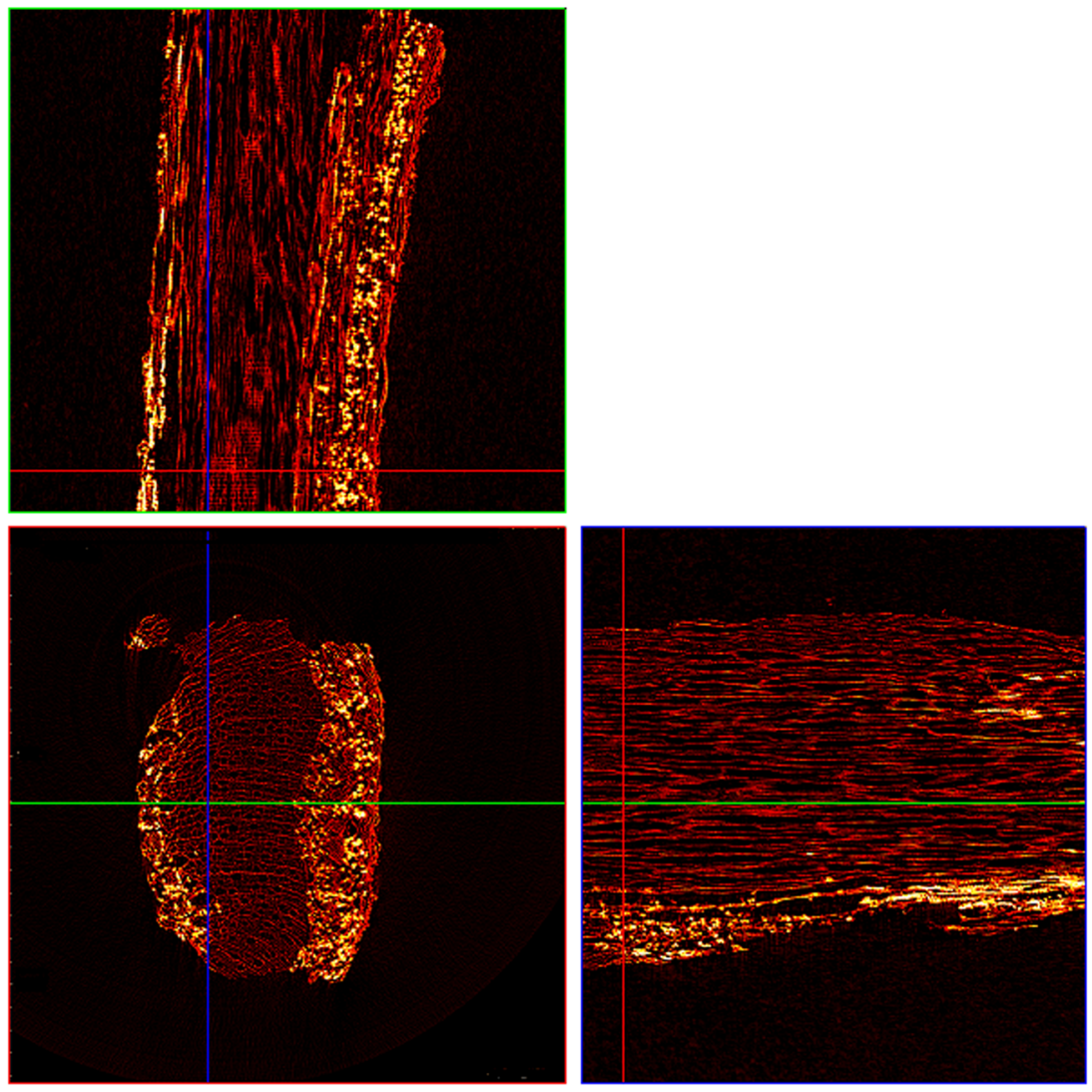

Figure 6. 3D crosshair view of an individual grain from the coarsest grained char fraction. Voxel resolution: $0.87 \mu \mathrm{m}$.

biochar in the following order of pore size homogeneity: $(1.0-2.0 \mathrm{~mm})<(0.3-1.0 \mathrm{~mm})<(<0.3 \mathrm{~mm})$. In other words, it is conceivable to hypothesize that the largest $1.0-2.0 \mathrm{~mm}$ fraction is made by larger biochar particles among which wide spaces exist where water can freely move. The surface of these biochar particles is made by a distribution of pores whose nominal size ranges from $<0.5$ up to $50 \mu \mathrm{m}$ according to the arbitrary classification reported in the preceding texts.

As biochar particle size decreases to $0.3-1.0 \mathrm{~mm}$, as a result of the use of a smaller stainless steel sieve, a higher packing density of sample particles is achieved. As a consequence, the transmissionlike pore spaces (having a nominal size of $\geq 50 \mu \mathrm{m}$ ) disappear to be replaced by a distribution of inter (i.e. among biochar particles) and intra (i.e. on particle surface) biochar particle spaces ranging from $<0.5$ up to $50 \mu \mathrm{m}$.

Further reduction to $<0.3 \mathrm{~mm}$ fraction increases biochar particle compaction, thereby providing a more homogeneous pore distribution with sizes from $<0.5 \mu \mathrm{m}$ up to the lower limit of the interval $0.5-50 \mu \mathrm{m}$.

\section{Nuclear magnetic resonance dispersion (NMRD) profiles}

Figure 2 reports the NMRD profiles (i.e. $R_{1}=1 / T_{1}$ values vs $\omega_{\mathrm{L}}$ ) of the water-saturated biochar fractions investigated here. The profiles are placed in the following $R_{1}$ order: $R_{1(1.0-2.0 \mathrm{~mm})}<R_{1(0.3-1.0 \mathrm{~mm})}<$ $R_{1(<0.3 \mathrm{~mm})}$. The latter behaviour accounts for the different pore size distribution in the three diverse textured biochar fractions. In fact, as previously indicated, fraction $1.0-2.0 \mathrm{~mm}$ is richer in large transmission-like pores that allow fast water motion, thereby leading to long proton longitudinal relaxation times and slower relaxation rates (refer to the relaxation mechanisms described in the preceding texts). Fraction $0.3-1.0 \mathrm{~mm}$ contains a larger amount of narrower storage-like and residual-like pores. For this reason, more water is bound to the pore walls at any given moment. As a consequence, stronger dipolar interactions result. The latter are responsible for shorter $T_{1}$ and faster $R_{1}$ values that place the NMRD profile above that retrieved for fraction $1.0-2.0 \mathrm{~mm}$. The last and more homogeneous $<0.3 \mathrm{~mm}$ fraction contains a larger amount of storagelike and residual-like pores than the other two fractions (refer in the preceding texts). Water molecules result, hence, even more immobilized than previously described. The fastest $R_{1}$ values are retrieved, and the profile placed on the top of Fig. 2 is acquired.

The qualitative evaluation of the NMRD profiles in Fig. 2 is also confirmed by their quantitative assessment through application of Eqns (2) to (3). Figure 3 shows the trend of three relaxometry parameters $\left(\alpha, \beta\right.$ and $\left.\left\langle\tau_{C}\right\rangle\right)$ for the water-saturated biochar fractions investigated here.

The average correlation time $\left\langle\tau_{C}\right\rangle$ as obtained by Eqn (3) is associated with the rate of molecular reorientation in the time unit. ${ }^{[21]}$ Namely, slower molecular motions, as a result of bound water molecules, produce larger $\left\langle\tau_{C}\right\rangle$ values, whereas shorter $\left\langle\tau_{C}\right\rangle$ values are related to fast molecular movements. Figure $3 \mathrm{~A}$ shows that correlation time is the shortest for the largest $1.0-2.0 \mathrm{~mm}$ fraction, intermediate for the fraction $0.3-1.0 \mathrm{~mm}$ and the longest for the finest $<0.3 \mathrm{~mm}$ fraction, thereby confirming that water motion is affected by the space available in each biochar fraction as previously indicated.

$\alpha$ is the relaxation rate at the high-field plateau of the NMRD profiles. ${ }^{[11]}$ The shorter the $\alpha$ value, the less constrained are the water molecules by the porous media resulting in weaker dipolar interactions. On the other hand, when water is tightly bound to biochar fractions, dipolar interactions become stronger and relaxation rates 

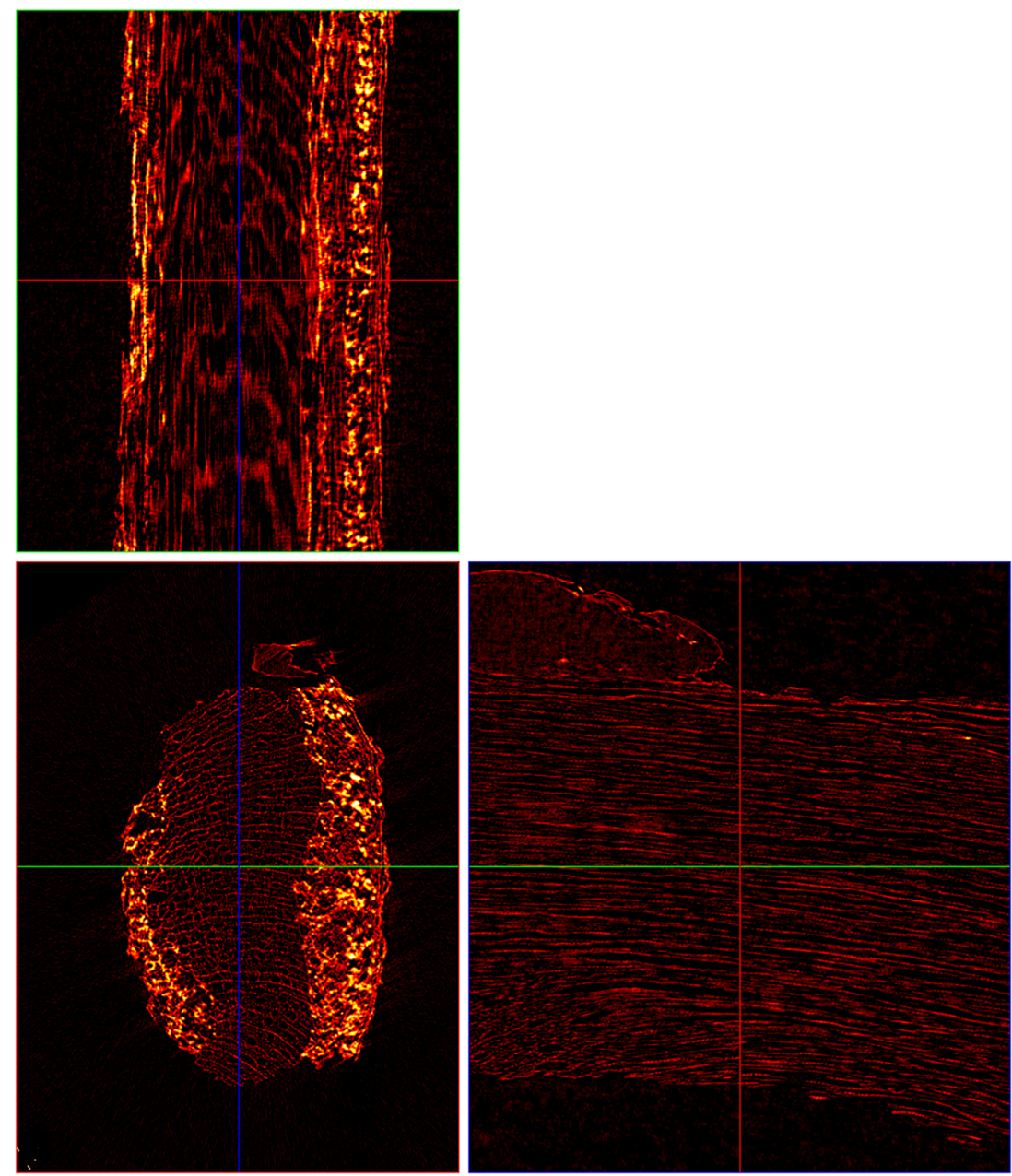

Figure 7. 3D crosshair view of a similar region of the same grain after water treatment for several days. The overall X-ray absorption of the regions with high inorganic content is reduced, but there are still substantial quantities of inorganic material present. Voxel resolution: $1.75 \mu \mathrm{m}$.

become faster. ${ }^{[21]}$ Figure 3B reports the $\alpha$ values for the three biochar texture fractions studied here. The smallest $\alpha$ value achieved for the coarsest $1.0-2.0 \mathrm{~mm}$ fraction confirms the fast water motion as a result of higher amount of large spaces available. The largest $\alpha$ value retrieved for $<0.3 \mathrm{~mm}$ fraction confirms that the space available for water motion is more restricted than in the other two fractions. Finally, the intermediate $\alpha$ value measured for fraction $0.3-$ $1.0 \mathrm{~mm}$ indicates that the space available for water movements is in between that present in fractions $1.0-2.0 \mathrm{~mm}$ and $<0.3 \mathrm{~mm}$.

$\beta$ is a measure of the force of the ${ }^{1} \mathrm{H}-{ }^{1} \mathrm{H}$ dipolar interactions. ${ }^{[15,22]}$ Increasing dipolar strengths, as a result of reduced water mobility, produce larger $\beta$ values. Conversely, weak dipolar couplings are generated by unbound (or freely moving) water, thereby providing smaller $\beta$ values. Once again, the results reported in Fig. $3 C$ confirm the expectations related to the space available for water motions. In fact, the smallest $\beta$ value obtained for the coarsest $1.0-2.0 \mathrm{~mm}$ biochar texture fraction indicates that water interacts weakly with pore walls as a result of its fast movements. The intermediate $\beta$ value achieved for fraction $0.3-1.0 \mathrm{~mm}$ suggests that biochar-water interactions are stronger than in $1.0-2.0 \mathrm{~mm}$ fraction but weaker than in $<0.3 \mathrm{~mm}$ one. Finally, the largest $\beta$ value retrieved for the finest $<0.3 \mathrm{~mm}$ fraction indicates that the dipolar interactions are the strongest because of the strong biochar-water interactions as a consequence of reduced mobility in restricted space.

\section{Micro-tomography results}

Figure 4 shows that there is a great variation in the packing density of the 1-2 mm sieve fraction of the biochar relative to the other fractions. This leads to a pronounced variation in the surface/volume ratio of the samples based on this type of biochar relative to the others. This lower surface/volume ratio is consistent with the much longer relaxation times reported for water in this sieve fraction relative to the other sieved fractions. In addition to the larger packing density of the finer sieve fractions, there is also a change in the inner pore structure of the char fragments. In the medium sieve fraction, there is still a substantial amount of biochar pieces with original wood pores in the range of several 10 to about $100 \mu \mathrm{m}$. Conversely, the finest fraction consists to a large extent of open fragments of wood-like pores and some grains with still intact wood-like porosity at a shorter length scale in the range of only a few micrometres (Fig. 5). This is mainly because of the fact that most of the larger wood pores are destroyed upon fragmentation in smaller pieces. Because of the increased fraction of open pores, the difference in surface/volume ratio between the medium and the fine grained sample is likely to be only moderate.

Additional insights into the nature and distribution of inorganic materials in the biochar material can be drawn from Figs. 6 and 7. While the centre of the grain consists essentially of well-preserved charred wood cells, the regions near the grain surface are richer 

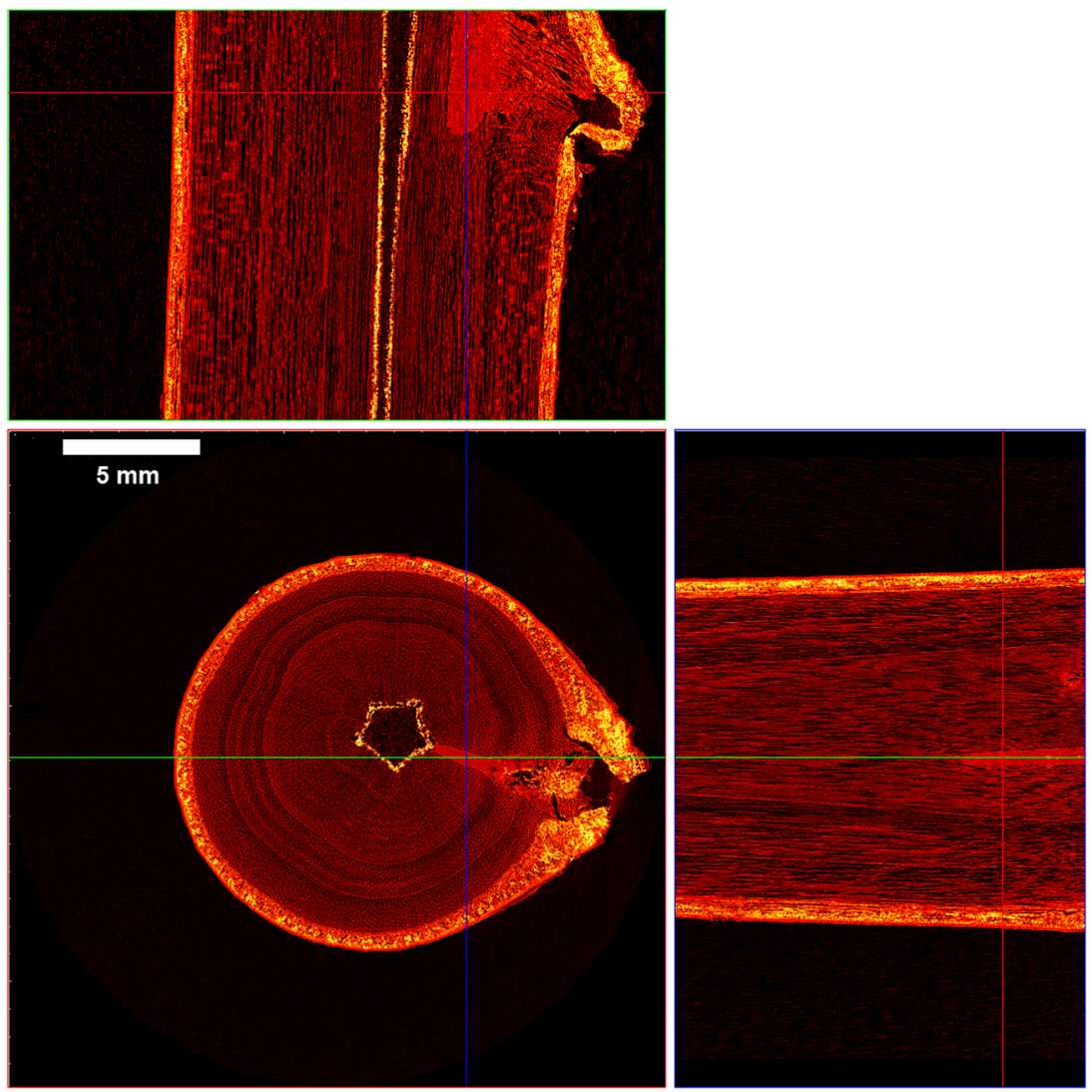

Figure 8. 3D crosshair view of a piece of a poplar twig in the typical size of the feedstock for the charring process. Voxel resolution: $5.98 \mu \mathrm{m}$.

in inorganic materials. These regions exhibit also a clear wood-like cellular structure. Like that, the inorganic load in these regions might be the result of an efflorescence-like accumulation of dissolved inorganic substances from the tissue water of the wood that migrates to these regions during the charring process. Alternatively, the inorganic material could be already inside these cells before the charring process. While parts of the inorganic material seem to be water soluble, another part of the inorganics still persists after water treatment over several days with a water volume about a thousand times larger than the volume of the char grain. Probably, a part of the inorganic material is calcined into poorly solvable materials during the charring process.

The scan of a fresh poplar twig in Fig. 8 indicates that in both near the bark and near the inner lumen of the twigs, there are considerable accumulations of inorganic materials. These latter are already present in the dried wood before charring. Whether these accumulations are the result of an efflorescence-like process during the drying of the wood or already present in the fresh wood needs to be addressed in a future study as well as a speciation of the inorganics.

\section{Conclusions}

The present study shows for the first time combination of X-ray micro-tomography and NMR relaxometry for the direct evaluation of the surface properties of three different biochars. These were selected by sieving an industrial poplar biochar with 1.0-2.0, 0.3-1.0 and $<0.3 \mathrm{~mm}$ stainless steel sieves.

The indirect NMR relaxometry probing revealed, as expected, that water dynamics is directly related to pore size distribution in different biochar textured fractions. In particular, the sole NMR relaxometry results confirmed previous understanding ${ }^{[7]}$ that predominance of 3D exchange between bound and bulk water may occur when the largest transmission-like pores are mainly present (the coarsest 1.0-2.0 $\mathrm{mm}$ fraction investigated in the present study). As inter-particle space reduces, as a result of the compaction following stainless steel sieving, progressive increment of the number of storage-like and residual-like pores is observed. According to these findings, we can speculate that water 2D diffusion can be mainly achieved as the amount of smallest pores is predominant (i.e. very large presence of residual-like pores and storage-like pores having a size included in the lower limit of the $0.5-50 \mu \mathrm{m}$ interval as in the $<0.3 \mathrm{~mm}$ fraction). Noteworthy, equal contribution of $2 \mathrm{D}$ and $3 \mathrm{D}$ diffusion may explain water motion in systems containing similar amounts of residual-like pores and storage-like pores such as in the fraction $0.3-1.0 \mathrm{~mm}$ used here.

The direct visualization of the pore structure by X-ray microtomography revealed the strong difference in packing density of the different sieved fractions and the presence of large quantities of multi-micron size porosity for all fractions. The larger extra-grain porosity and, correspondingly, the much lower surface/volume ratio for the largest sieve fraction confirmed the NMR relaxometry findings that the $3 \mathrm{D}$ water diffusion is the predominant mechanism for water motion in the coarsest 1.0-2.0 mm fraction. However, the large amount of multi-micron size porosity in all the fractions suggested that the 3D diffusion mechanism cannot even be neglected in the finest $<0.3 \mathrm{~mm}$ fraction. In fact, large amount of bulk water can be still present in multi-micron porosity, thereby suggesting that co-occurrence of 2D and 3D diffusion mechanisms result also in the finest biochar fraction.

An unexpected finding by X-ray micro-tomography was the very heterogeneous distribution of inorganic material inside all the 
biochar fractions. Part of those mineral components seem to be very poorly water-soluble, and therefore, they can be expected to contribute to water relaxation inside the char by their typically rather large surface relaxivities.

Future studies of biochar properties should take a closer look at the distribution and bio-availability of the inorganic components in different char materials as well as their impact on NMR relaxation behaviour of water in chars of different origin.

As there is already some evidence for the importance of inorganics on the effect of different types of biochar on plant growth, ${ }^{[23,24]}$ such studies are likely to provide novel mechanistic insights into these observations.

\section{Acknowledgements}

This study was partially funded by 'Progetto FFR 2102/2013' financed by Università degli Studi di Palermo. Experimental support in the micro-CT work by C. Willax and A. Sandor is gratefully acknowledged. Experimental support in NMR data acquisition by V. Marsala and G. Cimò, Università degli Studi di Palermo, is kindly acknowledged.

\section{References}

[1] D. Day, R. J. Evans, J. W. Lee, D. Reicosky. Energy 2005, 30, 2558-2579.

[2] B. Liang, J. Lehmann, D. Solomon, J. Kinyangi, J. Grossman, B. O'Neill, J. O. Skjemstad, J. Thies, F. J. Luizao, J. Petersen, E. G. Neves. Soil Sci. Soc. Am. J. 2006, 70, 1719-1730.

[3] S. P. Sohi, E. Krull, E. Lopez-Capel, R. Bol. Adv. Agron. 2010, 105, 47-82.
[4] M. Uchimiya, I. M. Lima, K. T. Klasson, S. Chang, L. H. Wartelle, J. E. Rodgers. J. Agr. Food Chem. 2010, 58, 5538-5544.

[5] J. H. Yuan, R. K. Xu, N. Wang, J. Y. Li. Pedosphere 2011, 21, 302-308.

[6] G. Baiamonte, C. De Pasquale, V. Marsala, G. Cimò, G. Alonzo, G. Crescimanno, P. Conte. J. Soils Sediments 2014. On-line-first DOI: 10.1007/s11368-014-0960-y

[7] P. Conte, U. M. Hanke, V. Marsala, G. Cimò, G. Alonzo, B. Glaser. J. Agric. Food Chem. 2014, 62, 4917-4923.

[8] P. Conte, V. Marsala, C. De Pasquale, S. Bubici, M. Valagussa, A. Pozzi, G. Alonzo. GCB Bioenergy 2013, 5, 116-121.

[9] C. De Pasquale, V. Marsala, A. E. Berns, M. Valagussa, A. Pozzi, G. Alonzo, P. Conte. J. Soil Sediments 2012, 12, 1211-1221.

[10] E. Anoardo, G. Galli, G. Ferrante. Appl. Magn. Reson. 2001, 20, 365-404.

[11] R. Kimmich, E. Anoardo. Prog. Nucl. Magn. Reson. Spectrosc. 2004, 44, 257-320.

[12] G. Ferrante, S. Sykora. Adv. Inorg. Chem. 2005, 57, 405-470.

[13] P. Conte, G. Alonzo. eMagRes 2013, 2, 389-398.

[14] L. A. Morozova-Roche, J. A. Jones, W. Noppe, C. M. Dobson. J. Mol. Biol. 1999, 289, 1056-1073.

[15] B. Halle, H. Johannesson, K. Venu. J. Magn. Reson. 1998, 135, 1-13.

[16] P. Conte, A. Maccotta, C. De Pasquale, S. Bubici, G. Alonzo. J. Agric. Food Sci. 2009, 57, 8748-8752.

[17] R. B. Clarkson, B. M. Odintsov, P. J. Ceroke, J. H. Ardenkjaer-Larsen, M. Fruianu, R. L. Belford. Phys. Med. Biol. 1998, 43, 1907-1920.

[18] S. Baronti, F. P. Vaccari, F. Miglietta, C. Calzolari, E. Lugato, S. Orlandini, R. Pini, C. Zulian, L. Genesio. Eur. J. Agron. 2014, 53, 38-44.

[19] A. G. Caporale, M. Pigna, A. Sommese, P. Conte. Biol. Fertility Soils 2014, 50, 1211-1222.

[20] K. R. Brownstein, C. E. Tarr. Phys. Rew. A 1979, 19, 2446-2454.

[21] V. I. Bakhmutov, Pratical NMR Relaxation for Chemists, John Wiley \& Sons Ltd, Chichester, West Sussex, UK, 2004.

[22] C. Luchinat, G. Parigi. Appl. Magn. Reson. 2008, 34, 379-392.

[23] H. Schulz, B. Glaser. J. Plant Nutr. Soil Sci. 2012, 175, 410-422.

[24] J. A. Fagbenro, S. O. Oshunsanya, O. A. Onawumi. Agrosearch 2013, 13, $57-68$. 